\title{
Electrostatic particle simulations of the WDL in the auroral plasma including the effects of up-flowing ions
}

\author{
A. Yajima and S. Machida \\ Department of Geophysics, Graduate School of Science, Kyoto University, Kyoto, Japan
}

(Received June 1, 2000; Revised November 9, 2000; Accepted November 9, 2000)

\begin{abstract}
An upward beam of hot ions which originates in the ionosphere is commonly observed in and above the region of auroral particle acceleration. Past simulations of the weak double layer (WDL) have only included magnetospheric hot electrons and ionospheric cold ions. We simulated the formation of the WDL numerically and more realistically using a one dimensional electrostatic code. We modeled magnetospheric hot electrons, ionospheric cold ions and hot ionospheric beam ions in the system. The existence of the hot ion beam modifies the characteristics of the WDL, its magnitude of the potential drop and lifetime. We investigated the variation in the character of the WDL for different fractions of the hot ion beam component. The magnitude of the potential jump of the WDL was found to decrease but the lifetime of the WDL increase as this proportion increased. The existence of a critical value of the ratio of the hot beam ions to the cold ions, above which electrons cannot be accelerated effectively and which places an upper limit on the altitude of auroral particle acceleration region has also been shown.
\end{abstract}

\section{Introduction}

Fast electrons are known to be created by field-aligned electrostatic potential drops over discrete auroral arcs. Electron energy-time diagrams obtained by satellite observation have an inverted-V structure due to this acceleration in the aurora (Frank and Ackerson, 1971). Simultaneous observation above a discrete arcs by Dynamics Explorer-I and II satellites showed that there was an electrostatic potential difference, aligned with the Earth's magnetic field between the two satellites (e.g., Reiff et al., 1988). Satellite observations have also revealed that the auroral particle acceleration region is in the range of altitudes from 1400 to $14000 \mathrm{~km}$ (Reiff et al., 1993).

It is thought that such electrostatic potential differences have a somewhat tenuous existence at first because charged particles can move easily along the magnetic field line and neutralize the charge layers which form the potential drop. Montgomery and Joyce (1969) and Knorr and Goertz (1974) found time-independent shockwave-like solutions in a system described by one-dimensional electrostatic VlasovPoisson equations. Later, the distribution of charged particles which can support the formation of a double layer was studied (e.g., Swift, 1975). However, such studies were concerned with steady state double layers. It is also necessary to develop time evolving models in order to study mechanisms which are responsible for the creation and development of double layers.

A number of types of computer simulation have been performed to study the formation of the double layer. They can be categorized into two basic types; potential-driven and

Copy right (C) The Society of Geomagnetism and Earth, Planetary and Space Sciences (SGEPSS); The Seismological Society of Japan; The Volcanological Society of Japan; The Geodetic Society of Japan; The Japanese Society for Planetary Sciences. current-driven. The current-driven type of simulation is suitable for studying the mechanism by which double layers are formed. For example, Sato and Okuda $(1980,1981)$ adopted modified boundary condition for their current-driven simulation. WDLs were formed in their simulation system as a result of the nonlinear growth of ion acoustic waves. The waves were excited by an electrostatic coupling between the stationary cold ions and hot electrons. It is still controversial that the WDLs actually accelerate the electrons enough to create aurora. However, it is extremely important to study the formation and characteristics of WDLs. A large-scale simulation of the whole region of auroral particle acceleration (Schriver and Ashour-Abdalla, 1993) has been carried out more recently. In the simulation, the field-aligned potential drop was formed by the charge separation which resulted from the difference of the mirror altitude between ions and electrons streaming towards the ionosphere (Alfvén and Fälthammar, 1963) and thus this is categorized to a different type from the WDL.

One theory of the WDL is closely related to the theory of ion holes. A negative electrostatic potential pulse $\left(e \phi_{0} / T \ll V_{t h}\right)$ is self-consistently maintained and exists with a hole in ion phase space (Dupree, 1982, 1983). The ion hole reflects the electrons in both sides of its boundaries. As these reflections are asymmetrical, a net potential jump can thereby be created (Berman et al., 1985). The Particle simulation has shown that the motion of ion holes in phase space can be equivalent to the motion of ordinary particles.

An electrostatic solitary wave in the auroral particle acceleration region was first reported by Temerin et al. (1982) followed by a larger number of observations by several other satellites. For example Koskinen et al. (1990) reported the observation of the solitary waves by the Viking satellite. Discrete arcs are formed in the upward current region of the au- 


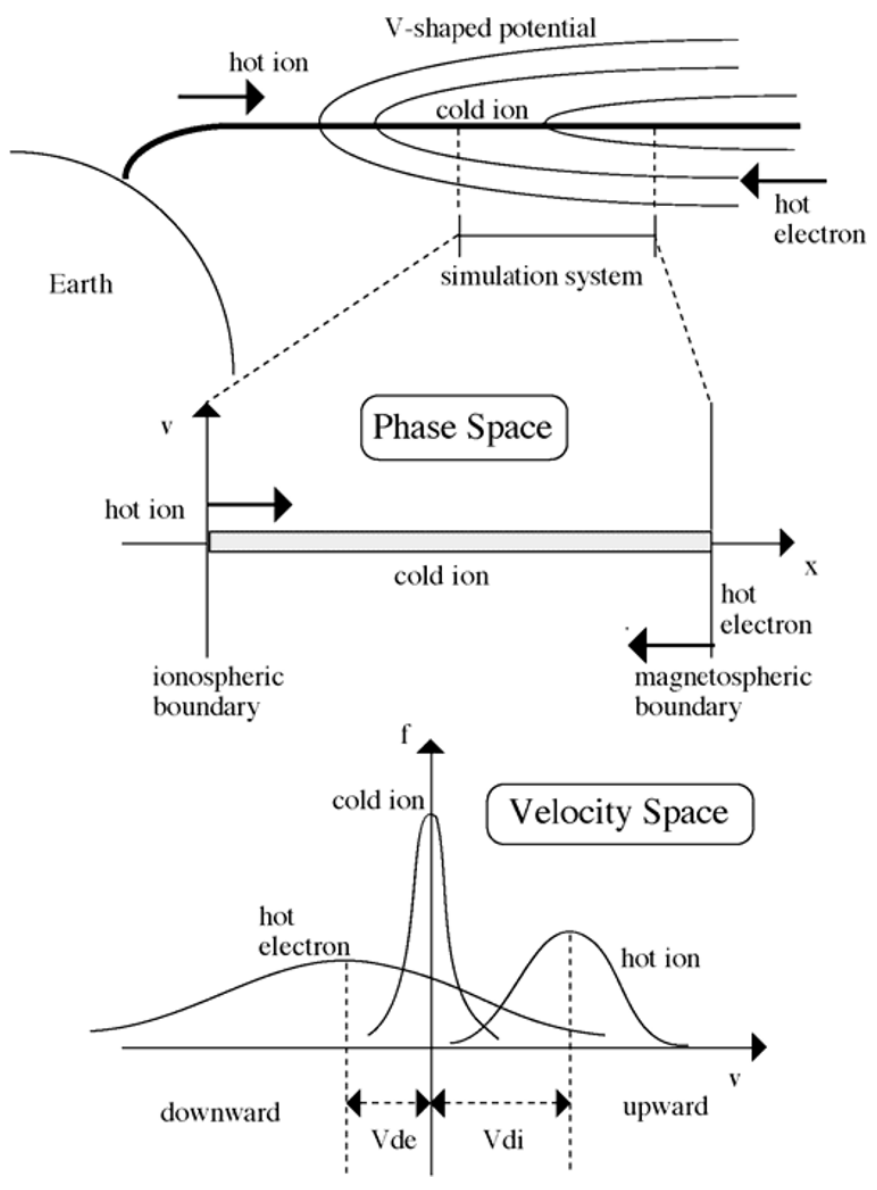

Fig. 1. A schematic diagram of the simulation model (top). The phase space of the simulation system which represents a portion of the auroral particle acceleration region (middle). The velocity distribution of the three components of the simulation system (bottom).

roral oval, and the upward field-aligned ion beam commonly exists in this region (Shelley et al., 1976; Reiff et al., 1988; Yamamoto et al., 1993). Cattell et al. (1998) calculated the plasma parameters in the auroral particle acceleration region with the particle and wave data obtained by FAST satellite. The density of each upward ion beam of $\mathrm{H}^{+}, \mathrm{O}^{+}$and $\mathrm{He}^{+}$was estimated to be $\geq 0.1 \mathrm{~cm}^{-3}$, while the total plasma density to be $\sim 1 \mathrm{~cm}^{-3}$. Then such hot ion beams are not negligible. Numerical simulations which only include ionospheric cold ions and magnetospheric hot electrons are not adequate to determine the mechanism that creates the potential drop. For this reason, our electrostatic particle simulation includes upward hot ions in addition to the ambient cold ions and magnetospheric hot electrons.

Originally a WDL is defined as the potential structure that composed of two charge layers with the potential jump less than the electron thermal energy. However, here we define that a WDL as a kind of solitary waves which have bipolar electric fields, i.e., solitary potential structures. We further define that a WDL is a developed solitary wave which has a potential jump comparable or slightly less than the electron thermal energy. Hence a WDL has three charge layers by definition. The ion hole is the depletion of the ions formed in their phase space density. It is known that the solitary wave of a negative pulse is projected to the ion hole in the ion phase space. We will discuss the acceleration of the WDL with those definitions.

\section{Simulation Model}

In this study, we numerically simulated two models, a three-component model and a two-component model. The latter has been done for the purpose of comparison. Both simulations were carried out with one dimensional electrostatic particle code in which an aperiodic boundary condition was adopted.

In the two-component model, we included hot shiftedMaxwellian electrons which carry the upward field-aligned current, and stationary cold ions which originate from the ionosphere. We set the temperatures and drift velocities of the ions and electrons to result in ion acoustic instability. The number of particles in the whole auroral acceleration region is too large to be handled by particle simulations, so we only simulated a portion of such an upward current region.

In the three-component model, we added an up-flowing ion beam component to those we had assumed for the twocomponent model. A field-aligned up-flowing ion beam is commonly observed in the auroral particle acceleration region by satellites, but this component has not been taken into account in previous work. Our simulations are schematically illustrated in Fig. 1.

We will present 10 cases. Run A and run F are of the twocomponent model, and the others are of the three-component 
model. The velocity distribution function of these components are as follows

$$
\begin{aligned}
f_{e}(v) & =n_{e}\left(m_{e} / 2 \pi T_{e}\right)^{1 / 2} \exp \left(-m_{e}\left(v-v_{d e}\right)^{2} / 2 T_{e}\right) \\
f_{c i}(v) & =n_{c i}\left(m_{i} / 2 \pi T_{c i}\right)^{1 / 2} \exp \left(-m_{i} v^{2} / 2 T_{c i}\right) \\
f_{h i}(v) & =n_{h i}\left(m_{i} / 2 \pi T_{h i}\right)^{1 / 2} \exp \left(-m_{i}\left(v-v_{d h i}\right)^{2} / 2 T_{h i}\right) \\
n_{e} & =n_{c i}+n_{h i} \quad m_{i} / m_{e}=100 \quad T_{e}=T_{h i}=20 T_{c i} .
\end{aligned}
$$

Parameters for all 10 cases are shown in Table 1 . There are three parameters in our simulation, the drift velocity of magnetospheric hot electrons $\left(V_{d e}\right)$, the ratio of the density of the hot ion beam component to all ionospheric ions $\left(N_{\text {hot }} / N_{\text {total }}\right)$, and the drift velocity of the hot ion beam $\left(V_{d h i}\right)$. According to Cattell et al. (1998), the total density of hot $\mathrm{H}^{+}, \mathrm{O}^{+}$and $\mathrm{He}^{+}$ion beams is $\sim 0.5 \mathrm{~cm}^{-3}$ and the total plasma density is $\sim 1 \mathrm{~cm}^{-3}$ in the auroral particle acceleration region. In our simulation, the maximum value of $N_{\text {hot }} / N_{\text {total }}$ is 0.5 , and this value can be regarded as being appropriate for the purpose of this simulation. The value of other parameters in our simulation is consistent with the result of Cattell et al., as well. We varied these parameters to adopt our model to different altitudes along the magnetic field line in the real auroral particle acceleration region. We executed the calculation with the parameter $N_{\text {hot }} / N_{\text {total }}=0.75$. However, we could not find the WDLs formed in this case, so we will not show the result for this.

We implemented the boundary conditions to achieve the followings, (1) A net potential drop across the simulation system is created as a result of the formation of WDLs. (2) The simulation system is connected to the constant current system. (3) The particles which are ejected and reinjected into the simulation system after accelerating do not affect the simulation system. Our model was set up as a charge-neutral system, so both boundaries should have equal electric field intensities. We fixed this value zero. We monitored the number of particles leaving the simulation system on every time step, and reinjected particles at both boundaries to keep a constant current density at both boundaries. The drift velocity of each component is calculated by averaging the particle velocities in the outermost grid, i.e., at the boundaries. With

Table 1. A summary of initial parameters: $V_{d e}$ (drift velocity of electrons) in units of $V_{t e}\left(=\sqrt{T_{e} / m_{e}}\right), V_{d h i}$ (drift velocity of hot ions) in units of $V_{t e}$ and $N_{h i} / N_{\text {total }}$ (ratio of hot ions to all ions). The other initial parameters, $T_{e} / T_{h i}=20$ and $m_{i} / m_{e}=100$, are common to all simulation runs.

\begin{tabular}{cccc}
\hline & $V_{d e} / V_{t e}$ & $V_{d h i} / V_{t e}$ & $N_{h i} / N_{\text {total }}$ \\
\hline run A & -0.4 & - & $0 \%$ \\
run B & -0.4 & 0.2 & $25 \%$ \\
run C & -0.4 & 0.3 & $25 \%$ \\
run D & -0.4 & 0.2 & $50 \%$ \\
run E & -0.4 & 0.3 & $50 \%$ \\
run F & -0.6 & - & $0 \%$ \\
run G & -0.6 & 0.2 & $25 \%$ \\
run H & -0.6 & 0.3 & $25 \%$ \\
run I & -0.6 & 0.2 & $50 \%$ \\
run J & -0.6 & 0.3 & $50 \%$ \\
\hline
\end{tabular}

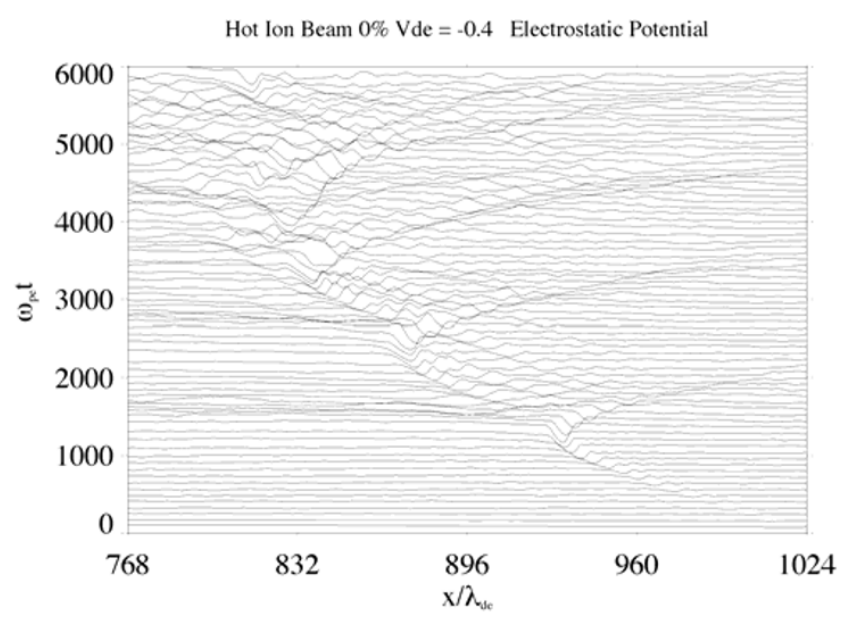

(a)

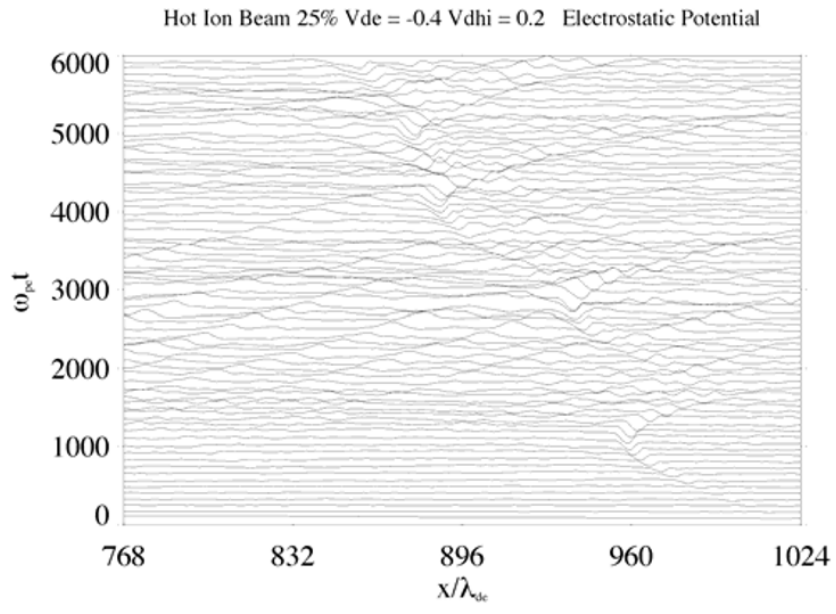

(b)

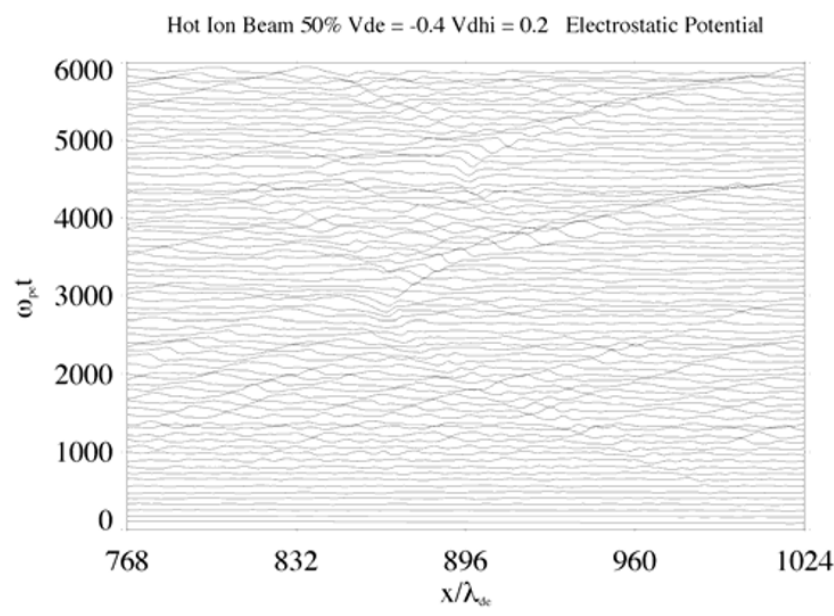

(c)

Fig. 2. Time-stacked plots of electrostatic potentials showing their development for three cases, with the fraction of hot ion beam of (a) $0 \%$, (b) $25 \%$ and (c) $50 \%$, respectively. 

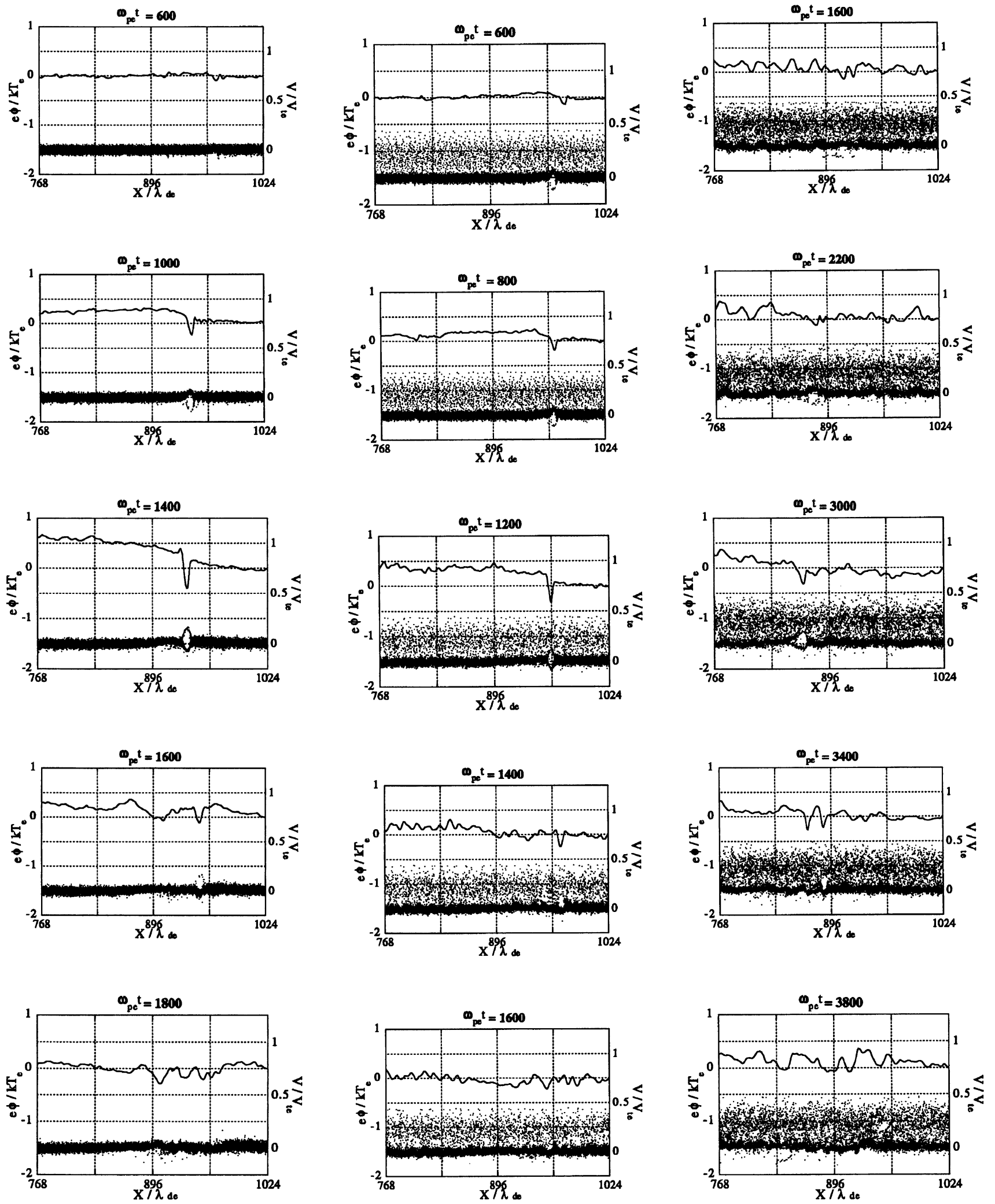

(a)

(b)

(c)

Fig. 3. Snapshot plots of electrostatic potentials and ion phase spaces, with the same simulation parameters as the respective cases in Fig. 2. 


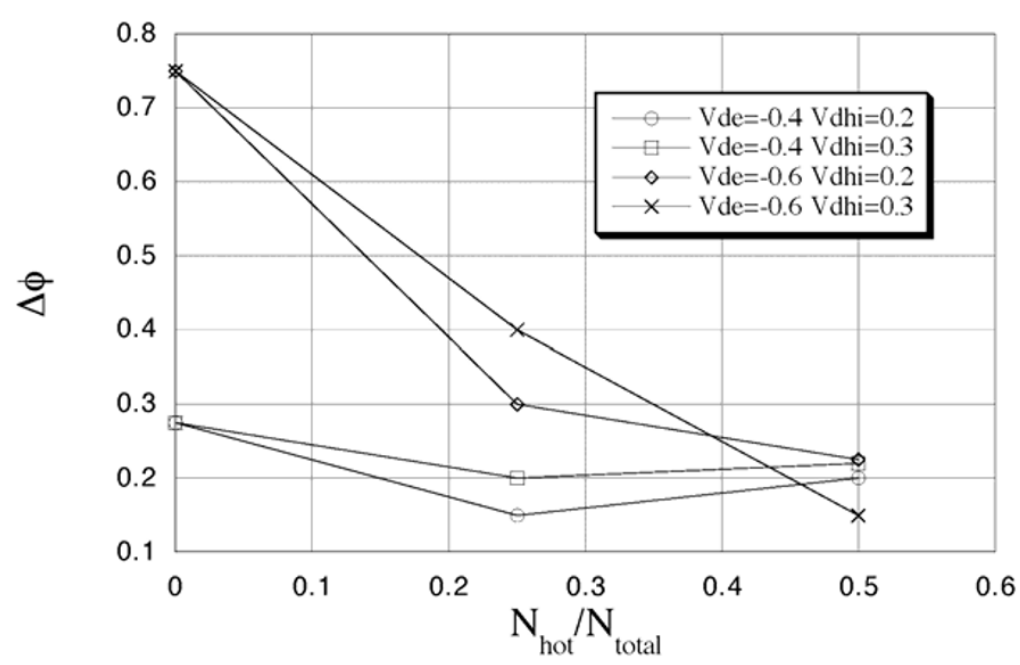

(a)

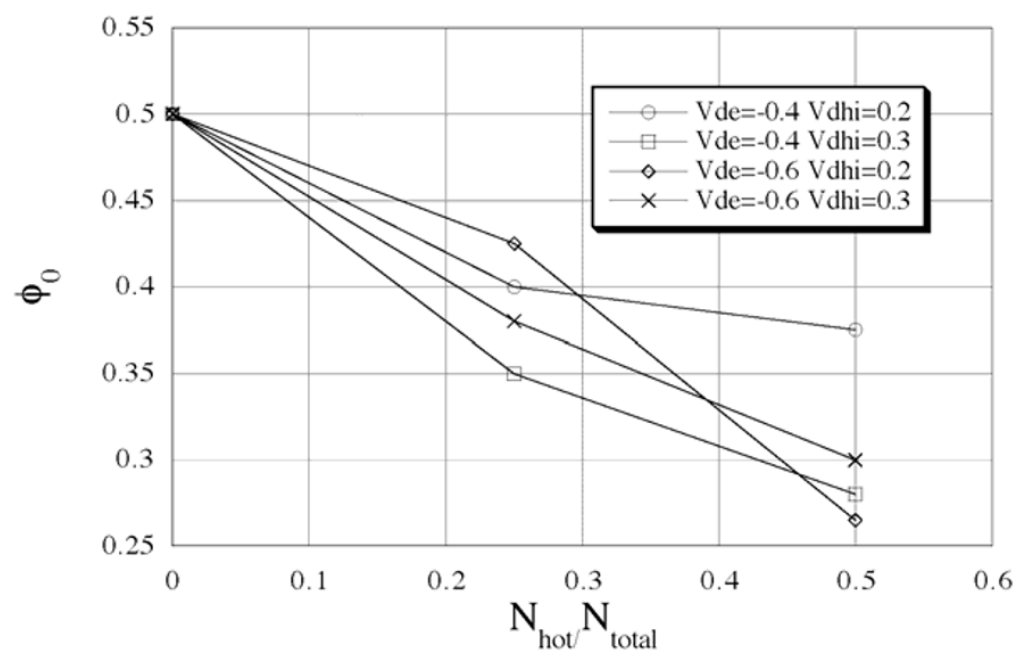

(b)

Fig. 4. Parameter dependence of the averaged potential jumps (a) $\Delta \phi$ and (b) $\phi_{0}$ of the WDL illustrated in Fig. 5.

our boundary condition, the boundaries do not affect the motion of particles that are accelerated and traveling outside the system.

\section{Simulation Results}

Figures 2(a), (b) and (c) are time-stacked plots of electrostatic potential, showing their development in three cases runs $\mathrm{A}, \mathrm{B}$ and $\mathrm{D}$ respectively (see Table 1 ). In all three cases, the WDL initially moves downward, in the same direction as the ion acoustic waves excited by the downward electron beam. At a certain time, the direction of motion of the WDL changes, and it starts to move upward, yielding the arc-like structure in these figures. This motion of the WDL is related to that of ion holes in phase space, as will be discussed later. The WDL goes through a repeated process of formation and dissipation, each of which results in the formation of a new WDL in a lower region. The lifetime of the WDL varies with the ratio of the density of hot ions to the overall density of ions. When all ions are cold, this process is repeated more than three times during the time interval from 0 to $4800 \omega_{p e}^{-1}$ as shown in Fig. 2(a). In contrast, it is repeated less than twice during the equivalent period when $50 \%$ of the ions are assumed to be cold as shown in Fig. 2(c). When a hot-ion component is included, new upward waves are excited along with the WDLs. The mode of these waves is different from that of the downward ion acoustic waves which create the WDLs.

In Figs. 3(a), (b) and (c) are snapshot plots of electrostatic potentials and ion phase spaces with the same simulation parameters as the respective parts of Fig. 2. Plots at five stages in the development of the WDL are shown for each case. In Fig. 2, the WDL displays an arc-like motion, and this is interpreted as being due to the acceleration of ion holes. The arc-like movement is divided into two phases, a solitary wave phase in which the WDL moves downward at a constant velocity, and an ion hole phase in which the WDL accelerates in the opposite direction and moves upward. The acceleration of the ion holes is attributed to the transfer of momentum from electrons. When the upward ion beams are present, ion holes continue to move in the ion beam region 
of phase space as shown in Fig. 3(c). The lifetime of the WDL becomes longer, as the fraction of the hot ion beams increases.

The WDL consists of three charge layers, i.e., positively, negatively and positively charged layers. The ion acoustic wave grows to become a solitary wave with a negative potential drop, and a three charge-layer structure. The solitary wave generated initially has no net potential jump. But, it has a field-aligned potential jump afterward, because the three charge layers have an asymmetric spatial structure. The negative potential well acts as a barrier against electrons, and electrons from both sides of the solitary wave are reflected by this potential barrier. Electrons stream downward along the magnetic field line faster than solitary waves, and more electrons are reflected in the upstream region than in the downward region. Due to the efficient reflection of electrons in the upstream region, the layer of positive charge on the upstream side of the three charge layers is weakened and contributes to a net potential jump.

Figures 4(a) and (b) show the parameter dependence of the averaged potential jumps $\Delta \phi$ and $\phi_{0}$ of WDLs as illustrated in Fig. 5. The four curves of each panel correspond to the results of our simulation with four different combinations of parameters. The value of $\Delta \phi$ decreases considerably as the fraction of hot beam-ions increases when the absolute value of $V_{d e}$ is large. The value of $\phi_{0}$ also decreases as the ratio of hot beam ions increases, but does not depend strongly on the value of $V_{d h i}$.

As will be explained later, the value of $\Delta \phi$ reaches the maximum when the drift velocity of hot electrons becomes equal to their thermal velocity. Therefore, $\Delta \phi$ is largest when $V_{d e}=-0.6$ in Fig. 4(a). Figure 4(b) clearly shows the decrease in $\phi_{0}$ with the fraction of hot beam-ion. The fraction of hot beam-ions is the parameter with the most effect on the net potential jump of a WDL.

The magnitude of $\Delta \phi$ is dependent on the magnitude of $\phi_{0}$, because more electrons are reflected by the electrostatic potential barrier and the charge layers thus take on a more asymmetric structure. This is consistent with the fact that the decrease in $\Delta \phi$ with the fraction of hot beam-ions occurs simultaneously with the decrease in $\phi_{0}$, as shown in Figs. 4(a) and (b). When $N_{\text {hot }} / N_{\text {total }}$ is 0.5 , the magnitudes of $\Delta \phi$ are no longer decreasing much effectively except for the case with $V_{d e}=-0.6$ and $V_{d h i}=0.3$. We interpret this as that the hot ion beam affects the formation of the WDL through the excitation of additional electrostatic waves, and our simple

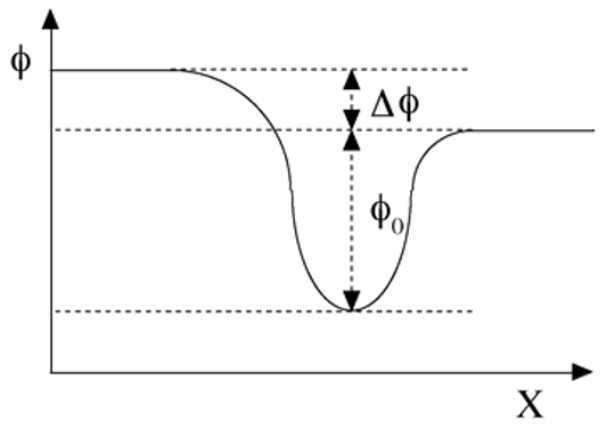

Fig. 5. Potential profile of a WDL. ion hole theory is not applicable to these.

In Figs. 2(a), (b) and (c), the velocity of the solitary wave varies with the proportion of hot ions. The WDL is a consequence of the interaction between the solitary wave and cold ions, hence, this value is thought to affect the process of WDL formation. Figures 6(a) and (b) show the relationship between the depth of the potential well $\left(\phi_{0}\right)$ and the velocity of the solitary wave resulting in the WDL. The velocity of the solitary wave was derived from the results of simulation. $\left(V_{s w}-V_{t i}\right)^{2}$ is useful for examining the velocity of the solitary wave in view of its role in the formation of the WDL, because its square root indicates the magnitude of the solitary wave and $V_{s w}-V_{t i}$ indicates the distance in phase space between the solitary wave and cold ions.

Both $\left(V_{s w}-V_{t i}\right)^{2}$ and $\phi_{0}$ are decreased when the contribution of the hot-ion beam increases. The hot-ion beam decelerates the velocity of the solitary wave, and this decreases the depth of the potential well, as will be explained in the following section.

Figure 7(a) illustrates the development of the WDL. The five ellipses show electrostatic potential structures induced by the solitary wave which correspond to ion holes in the $x-V$ plane of ions, at five different time steps. The vertical size of an ion hole can be interpreted as the depth of a negative potential pulse. The development of the WDL over time takes place in two stages, i.e., a stage characterized by solitarywave motion and an ion-hole like motion. During the stage of solitary wave motion ( $t=t_{1}, t_{2}$ and $\left.t_{3}\right)$, the electrostatic potential of the solitary wave grows and moves to the left. The growth of the solitary wave reaches saturation due to the interaction between the ion hole and cold ions $\left(t=t_{4}\right)$, followed by the next stage in which the WDL moves in a similar way to an ion hole $\left(t=t_{5}\right)$. The amplitude of the solitary wave stays constant during this stage. The magnitude of the ion hole depends on the difference $(\Delta V)$ between the velocity of the solitary wave $\left(V_{s w}\right)$ and the thermal velocity of cold ions $\left(V_{t h}\right)$. The solitary wave can grow until its trapping boundary meets with the cold ions in phase space. Therefore, the potential jump $\phi_{0}$ of WDL can grow to approximately $m_{i} \Delta V^{2} / 2 e$. Figure 7(b) shows the motion of the WDL formed in run $\mathrm{B}$ with the same simulation parameters to the case shown in Figs. 2(b) and 3(b). The curve illustrated in this figure represents the arc-like motion of the WDL. To show the location of the WDL, the local minimum of the electrostatic potential is marked. The solid curve shows the parabolic fitting. We regard the motion of the WDL as a uniformly accelerated motion during the ion hole like motion of the WDL from $\omega_{p e} t=600$ to $\omega_{p e} t=1400$. We sampled the phase space scatter plot (Fig. 3) every $\omega_{p e} t=200$. Examining the growth of the WDL in the view of the ion phase space, we found that $\omega_{p e} t=600$ is a proper time to divide the two stages. In Fig. 3(b), $\omega_{p e} t=600$ is the time when ions distribute around the ion hole, i.e., slightly after a trapping boundary of the ion hole meets with the cold ions in the phase space. A solitary wave of the electrostatic potential can be projected to an ion hole on the phase space as shown in Fig. 7(a). The ion hole keeps uniformly acceleration motion with the momentum exchange of electrons as a rigid body. The WDL is given momentum and energy by electrons to enlarge the amplitude in the first stage. In the second stage, 


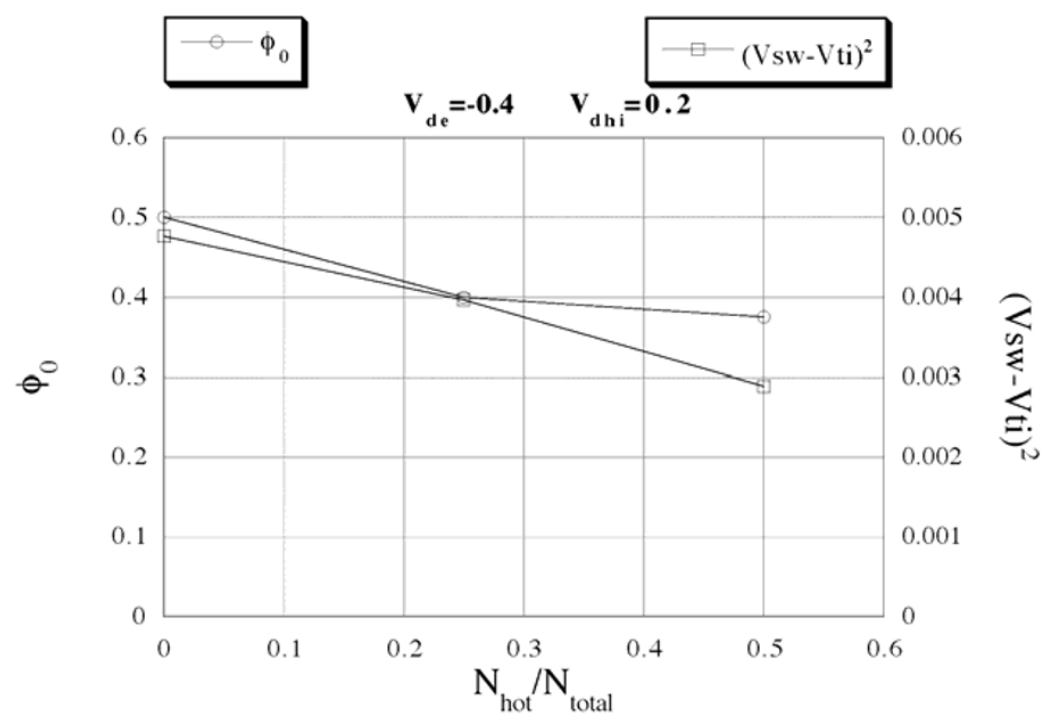

(a)

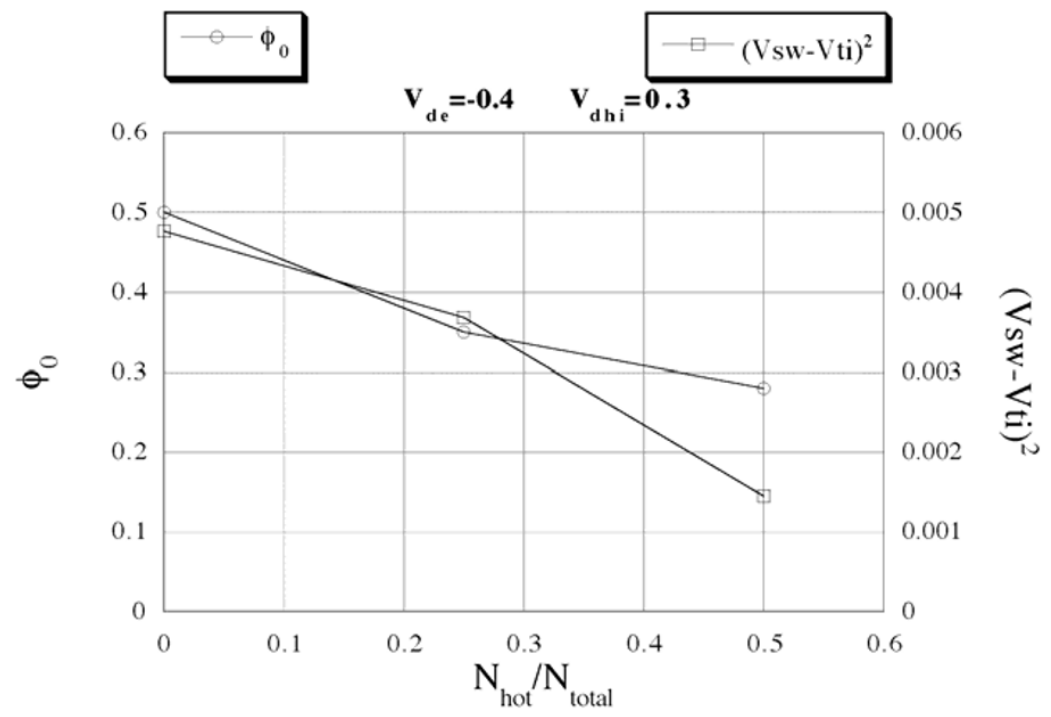

(b)

Fig. 6. Relationship between the depth of the potential well $\left(\phi_{0}\right)$ and the velocity of the solitary wave which produces the WDL (a) in the case of $V_{d e}=-0.4$, $V_{d h i}=0.2$, and (b) in the case of $V_{d e}=-0.4, V_{d h i}=0.3$. The velocity of the solitary wave was obtained from the result of simulation.

the ion hole behaves as a rigid body and is accelerated exchanging momentum with electrons (Dupree, 1983; Berman et al., 1985).

Figure 8 shows a linear dispersion relation ( $\omega-k$ diagram) of ion acoustic waves for three cases which correspond to the curve of $V_{d e}=-0.4$ and $V_{d h i}=0.2$ in Fig. 4 . As can be seen in the figure, the phase velocity of the mode with the highest growth rate decreases with an increasing fraction of hot ions, namely, $\omega / k=0.0674,0.0615$ and 0.0533 at $k=0.905$, 0.845 and 0.750 for $N_{\text {hot }} / N_{\text {total }}=0.00$ (a), 0.25 (b) and 0.50 (c), respectively. The same tendency can be found in the other cases in which we included the hot ion component.

\section{Discussion}

In our simulation, the WDL lasts longer when there is a greater proportion of hot ions. There are two reasons for this. First, the transfer of momentum between the ion hole and hot electrons decreases when $\phi_{0}$ is small, and the depth of potential well determines the range of velocity where the hot electrons are reflected by the potential barrier. The acceleration of an ion hole is due to this transfer of momentum, and its lifetime is short when it is accelerated rapidly.

An ideal ion hole has a size in the ion phase space less than the thermal velocity of the background ions, and has characteristics of a rigid body. Therefore one difference between the ion hole formed in the present work and an ideal ion hole is the size of the hole in ion phase space. Our WDL is accompanied by the ion hole whose size is comparable to the thermal velocity of ions. However, the basic physics involved in both phenomena are the same. We will show 


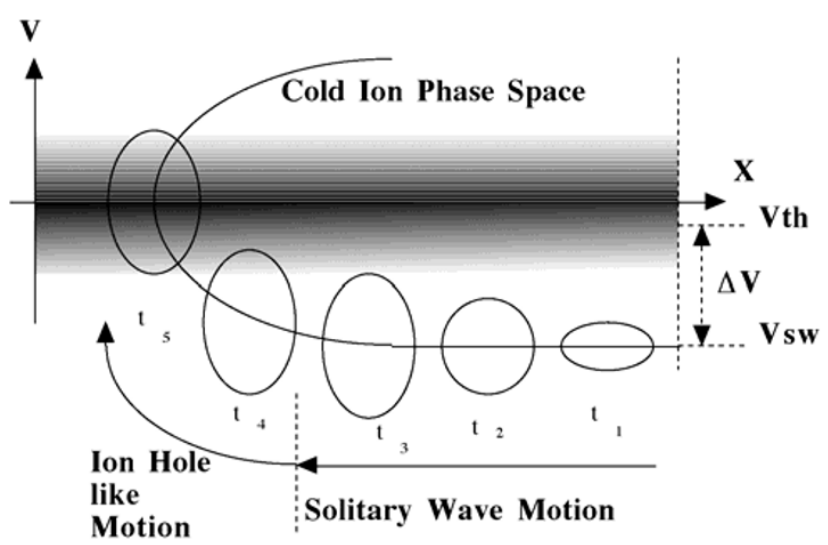

(a)

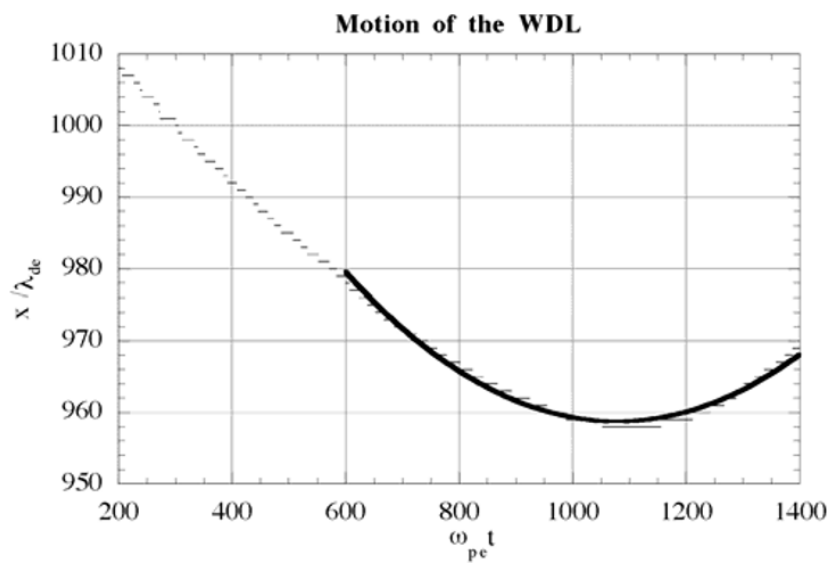

(b)

Fig. 7. (a) Development of the WDL. The five ellipses show electrostatic potential structures induced by the solitary wave which correspond to ion holes in the $x-V$ phase space of ions, at five time steps. The vertical size of an ion hole can be interpreted as the depth of a negative potential pulse. (b) Motion of the WDL. To show the location of the WDL, the local minimum of the electrostatic potential is marked. The solid curve shows the fitted motion of the WDL.

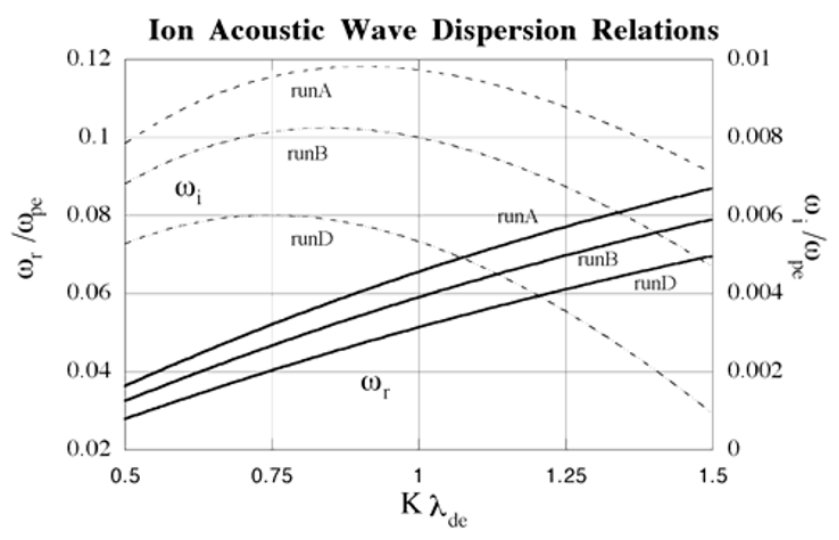

Fig. 8. A linear dispersion relation ( $\omega-k$ diagram) of ion acoustic waves, for three cases which correspond to the curve for $V_{d e}=-0.4$ and $V_{d h i}=0.2$ in Fig. 4. that the lifetime of our WDL can be estimated as an ideal ion hole.

The acceleration of the ion hole is calculated with the assumption that momentum is transferred between electrons and an ion hole as if the hole were rigid body (Berman et al., 1985). Thus, the lifetime of the WDL can also be estimated by a similar momentum-transfer argument. In the downward region of the WDL, electrons are reflected and gain momentum, and electrons are reflected in a similar way in the upstream region with a momentum loss, because the value of $f_{e}^{\prime}\left(V_{\mathrm{WDL}}\right)$ is not equal to zero. The electrons reflected from both sides of the WDL can gain $m \Delta x^{-1} \Delta v^{4} f_{e}^{\prime}\left(V_{\mathrm{WDL}}\right)$ of momentum per unit time, where $\Delta V\left(=\sqrt{2 e \phi_{0} / m}\right)$ is the range of the velocity of electrons reflected by the potential barrier $\phi_{0}$, and $\Delta x$ is the size of the WDL in real space (Berman et al., 1985; Tetreault, 1991).

Considering the inertial term of the equation of motion of an ion hole, the ion hole can be assumed to contain a negative mass in the form of its background ions. The magnitude $\phi_{0}$ of the WDL increases until formation of the ion hole is complete. The depletion of ion density in the WDL cannot be calculated analytically, and the velocity distribution function of background ions is modified by the WDL. By approximating the shape of the potential field of the WDL as $\phi(x)=\phi_{0}\left(1-(2 x / \Delta x)^{2}\right)$, and noting that the distribution of ions is proportional to this approximated potential as concluded from theory (Mälkki et al., 1989), we estimated the pseudo-mass of the WDL from the results of our simulations. We obtained a pseudo-mass of $\int \Delta n_{0}\left(1-(2 x / \Delta x)^{2}\right) d x$. The equation of motion of a WDL is then (4/3) $M \Delta n_{0} \Delta x V_{\mathrm{WDL}}^{\prime}=$ $m \Delta x^{-1} \Delta v^{4} f_{e}^{\prime}\left(V_{\mathrm{WDL}}\right)$, where $\Delta n_{0}$ is the maximum depletion of density. The lifetime of a WDL can be defined as the period over which the velocity of the WDL changes from $-V_{i t h}$ to $V_{i t h}$. The magnitude of $\phi_{0}$ and the efficiency with which a WDL is accelerated decrease with the proportion of hot ions. In our three cases $N_{\text {hot }} / N_{\text {total }}=0.00,0.25$ and 0.50 with common parameters $V_{d e}=-0.4$ and $V_{d h i}=0.2$ as shown in Fig. 6(a), the lifetime of the WDLs were evaluated as $1.3 \times 10^{3} \omega_{p e}^{-1}, 2.1 \times 10^{3} \omega_{p e}^{-1}$, and $2.4 \times 10^{3} \omega_{p e}^{-1}$. This roughly agrees with the results of our simulation in Fig. 2. In Fig. 7(b), we assumed that the acceleration of the WDL is constant, and the obtained acceleration rate is $1.85 \times 10^{-5} \lambda_{d e} \omega_{p e}^{2}$. This agrees with the value derived from the equation of motion mentioned above.

Secondly, the WDL moves into the hot ion beam in ion phase space. In the two-component model, the WDL disappears after traveling across the cold ions in ion phase space. When there are hot ions, however, the WDL can move further into the hot ions in phase space. Of course, the existence of an upward flow of hot ions does not necessarily ensure that the lifetime of a WDL will be prolonged. When $V_{d h i}$ is much greater than the size of the ion hole in ion phase space, the WDL cannot move into the hot ion region, because the WDL cannot exist without background ions. The background ions are distributed broadly throughout ion phase space, when the hot ions have a high thermal velocity. The shape of the distribution function of hot ions thus affects the lifetime of WDL.

There is another interesting topic to do with the rate of acceleration and lifetime of the WDL, and that is the formation 
of the strong double layer (SDL). The ion hole is striking in that it behaves as if it were a robust object against real ions, and the ions move along the outermost boundary of the ion hole in phase space. However, this assumption is not valid, when the ion hole is accelerating rapidly. In such a case, the distribution function of background cold ions cannot recover to its initial unperturbed state after being crossed by the ion hole. We found in our simulation that this is closely related to the formation of the SDL. We would like to devote some future study to this phenomenon.

In Figs. 2(b) and (c), upward waves are excited additionally along with downward waves. These upward waves create a characteristic electrostatic potential structure as well as the downward ion acoustic wave. The potential structure created by the upward wave contributes to the acceleration of electrons. We estimated the velocity of a solitary wave moving downward which developed from the ion acoustic waves in Fig. 2. We adopted initial conditions which were intended to excite the ion acoustic waves. However, during linear growth of the wave, the motion of the ion acoustic wave is not visible in Fig. 2 because of its very low amplitude.

The magnitude of $\phi_{0}$ depends on the velocity of the solitary wave, and therefore only indirectly upon the velocity of the ion acoustic wave. Figure 8 shows curves on $\omega-k$ diagrams which represent the ion acoustic waves in their linear growth phase. The solitary wave is faster than the ion acoustic wave, and the reduction of the velocity of solitary wave with an increasing proportion of hot ions is similar to the behavior ion acoustic wave in its linear-growth phase, because the solitary wave is the consequence of the nonlinear growth of ion acoustic wave.

\section{Summary and Conclusion}

We simulated the formation of the WDL numerically, changing the ratio of the upward ion beam component to the total amount of ions as a simulation parameter.

The profile of the WDL varies with the proportion of hot ion beam. In particular, the value of $\Delta \phi$ decreases as this proportion increases. The size of $\Delta \phi$ depends on the depth of the potential well $\left(\phi_{0}\right)$, by which hot electrons are reflected to form the asymmetrical structure of the WDL with three charge layers. $\phi_{0}$ is equivalent to the amplitude of the solitary wave which is a consequence of the ion acoustic wave. This mode is excited under the cold ions and the hot shiftedMaxwellian electrons which supply energy to the wave. Ion acoustic wave continues to grow to form a solitary wave. The solitary wave stops growing when the wave begins to interact with the cold ions. In ion phase space, the ion hole, to which the solitary wave corresponds, grows until its boundary reaches into the region of cold ions. The cold ions begin to be distributed around the ion hole, and the ion hole becomes visible. Taking the process noted above into account, the velocity difference between the solitary wave and cold ions in ion phase space is important in determing the size of $\phi_{0}$. Specifically, the velocity of the solitary wave $\left(V_{s w}\right)$ is significant. The results of simulation shows the strong dependence of $\phi_{0}$ on $V_{s w}$. As $\phi_{0}$ decreases with the fraction of hot ion beam, $V_{s w}$ tend to decrease with ion beams.

In the real auroral particle acceleration region, the value of $N_{\text {hot }} / N_{\text {total }}$ increases with the altitude, because the cold ions are not accelerated by WDLs though the hot ions are accelerated and travel upward. In our simulation, the value of $N_{\text {hot }} / N_{\text {total }}$ is the key parameter of the existence of WDL. It means that this value determines the altitude of upper boundary of the auroral particle acceleration region. In the lower altitudes, the value of $N_{\text {hot }} / N_{\text {total }}$ is adequate to form WDLs, and furthermore the lifetime of WDL is long when the drift velocity of the hot ion beam is not so large. Thus the potential structure in the auroral particle acceleration region must be controlled by the value of $N_{\text {hot }} / N_{\text {total }}$.

Acknowledgments. The author expresses gratitude to Prof. Tohru Araki and Dr. Akinori Saitou of the Kyoto University, Prof. Toshihiko Iyemori, and Drs. Toyohisa Kamei and Masahiko Takeda of the World Data Center C2 for Geomagnetism, Kyoto University. Valuable comments in the seminars were very useful to conduct this research. The author thanks all the colleagues in the Solar-Planetary Electromagnetism Labratory, Graduate School of Science, Kyoto University. He was able to have a number of invaluable suggestions and comments from them.

\section{References}

Alfvén, H. and C.-G. Fälthammar, Cosmical Electrodynamics, Fundamental Principles, pp. 161-167, Oxford Univ. Press, New York, 1963.

Berman, R. H., D. J. Tetreault, and T. H. Dupree, Simulation of phase space hole growth and the development of intermittent plasma turbulence, Phys. Fluids, 28, 155-176, 1985.

Cattell, C., et al., The association of electrostatic ion cyclotron waves, ion and electron beams and field-aligned currents: FAST observations of an auroral zone crossing near midnight, Geophys. Res. Lett., 25, 2053-2056, 1998.

Dupree, T. H., Theory of phase-space density holes, Phys. Fluids, 25, 277289, 1982.

Dupree, T. H., Growth of phase-space density holes, Phys. Fluids, 26, 24602481, 1983.

Frank, L. A. and K. L. Ackerson, Observations of charged particle precipitation into the auroral zone, J. Geophys. Res., 76, 3612-3643, 1971.

Knorr, G. and C. K. Goertz, Existence and stability of strong potential double layers, Astrophys. Space Sci., 31, 209-223, 1974.

Koskinen, H. J., et al., On the plasma environment of solitary waves and weak double layers, J. Geophys. Res., 95, 5921-5929, 1990.

Mälkki, A., et al., On theories attempting to explain observations of solitary waves and weak double layers in the auroral magnetosphere, Phys. Scr., 39, 787-793, 1989.

Montgomery, D. and G. Joyce, Shock-like solutions of the electrostatic Vlasov equation, J. Plasma Physics, 3, 1-11, 1969.

Reiff, P. H., et al., Determination of auroral electrostatic potentials using high- and low-altitude particle distributions, J. Geophys. Res., 93, 74417465,1988

Reiff, P. H., et al., On the high- and low-altitude limits of the auroral electric field region, J. Auroral Plasma Dynamics Geophysical Monograph, 80, 143-154, 1993.

Sato, T. and H. Okuda, Ion acoustic double layers, Phys. Rev. Lett., 44, 740-743, 1980.

Sato, T. and H. Okuda, Numerical simulations on ion accoustic double layers, J. Geophys. Res., 86, 3357-3368, 1981.

Schriver, D. and M. Ashour-Abdalla, Self-consistent formation of parallel electric fields in the auroral zone, Geophys. Res. Lett., 20, 475-478, 1993.

Shelley, E. G., et al., Satellite observations of an ionospheric acceleration mechanism, Geophys. Res. Lett., 3, 654-656, 1976.

Swift, D. W., On the formation of auroral arcs and acceleration of auroral electrons, J. Geophys. Res., 80, 2096-2108, 1975.

Temerin, M., et al., Observations of double layers and solitary waves in the auroral plasma, Phys. Rev. Lett., 48, 1175-1179, 1982.

Tetreault, D. J., Theory of electric fields in the auroral acceleration region, J. Geophys. Res., 96, 3549-3563, 1991.

Yamamoto, T., et al., Meridional structures of electric potentials relevant to premidnight discrete auroras: A case study from Akebono measurements, J. Geophys. Res., 98, 11,135-11,151, 1993.

A. Yajima (e-mail: yajima@kugi.kyoto-u.ac.jp) and S. Machida 